\title{
An adaptive granular RVE model with an evolutionary periodic boundary for hierarchical multiscale analysis
}

\author{
T.M. Qu ${ }^{1}$, Y.T. Feng ${ }^{1}$, Min Wang ${ }^{2 *}$
}

1 Zienkiewicz Centre for Computational Engineering, College of Engineering, Swansea University, Swansea, Wales, SA1 8EP, UK

2 T-3 Fluid Dynamics and Solid Mechanics Group, Theoretical Division, Los Alamos National Laboratory, Los Alamos, New Mexico 87545, USA

* corresponding author: minw@lanl.gov or sacewangmin@gmail.com

\begin{abstract}
:
The hierarchical multiscale analysis normally utilises a microscopic representative volume element (RVE) model to capture path/history-dependent macroscopic responses instead of using phenomenological constitutive models. However, for problems involving large deformation, the current RVE model used in geomechanics may lose representative properties due to the progressive distortion of the RVE box, unless a proper reinitialization is applied. This work develops an adaptive RVE model in conjunction with an evolutionary periodic boundary (EPB) algorithm for hierarchical multiscale analysis of granular materials undergoing large deformation based on a recent RVE model proposed for coupling molecular dynamics and the material point method. The proposed adaptive RVE model avoids the reinitialization of the RVE box that even undergoes extremely large shear deformation; meanwhile, it accounts for the deformation history of the RVE model and treats the interaction between boundary particles and other image particles in a more efficient way. Numerical examples with extremely large deformation are used to illustrate the adaptive granular RVE model enhanced by the proposed EPB algorithm. Furthermore, some key features of this new methodology are further discussed for clarification.
\end{abstract}




\section{Keywords:}

Multiscale analysis; Granular material; Adaptive representative volume element; Discrete element method; Evolutionary periodic boundary.

\section{Introduction}

Granular materials are commonly encountered in geotechnical engineering, where large deformation processes, such as slope failure, happen from time to time. Current constitutive models are limited in accurately predicting large deformation of granular geomaterials. Over the past fifteen years, efforts have been made to study the physics of granular materials and simulate their mechanical behaviour using multiscale numerical tools, such as the combination of the discrete element method (DEM) with the continuum-based finite element method (FEM), the finite difference method (FDM) and the material point method (MPM) ${ }^{1-4}$.

Two primary classes of multiscale models in geomechanics can be found in the literature ${ }^{5,6}$. One is called the concurrent approach where the entire domain is partitioned into a discrete subdomain (simulated by DEM) and a continuum subdomain (simulated by FEM or FDM) ${ }^{1,2,7}$. The nature of concurrent multiscale models is that both the discrete subdomain and the continuum subdomain are independently solved in a single timestep but coupled at their interfaces; i.e. the two subdomains act as dynamic boundary conditions for each other. The other class of multiscale models is called the hierarchical approach where the whole model is simulated by macroscopic methods (e.g. FEM or MPM), but instead of using the phenomenological constitutive relations, the constitutive laws are provided by the microscopic modelling (e.g. DEM) in conjunction with some homogenization schemes.

The current study falls into the hierarchical multiscale modelling approach. The early work of hierarchical multiscale modelling in geomechanics can be traced back to the literature ${ }^{8-13}$. Andrade and $\mathrm{Tu}{ }^{14,15}$ proposed a multiscale method in the modelling of granular materials. This method performs grain-scale computations by DEM to obtain 
the evolution of basic plastic variables and then passes the data to continuum computations by FEM. Later, Miehe et al. ${ }^{13}$, Nitka et al. ${ }^{16,17}$, Nguyen et al. ${ }^{18}$, and Guo and Zhao ${ }^{19}$ developed conceptually similar multiscale approaches in which FEM is used to simulate the macroscopic deformation while the granular RVE modelled by DEM is utilised to consider grain interactions at the micro-level. The computational homogenisation is used to derive the constitutive relations based on the numerical responses of the DEM model at each Gauss point of FEM and periodic boundary conditions (PBC) are applied to the RVE to eliminate boundary effects. More extended applications with a similar hierarchical multiscale technique to geomechanics were reported in ${ }^{20-28}$. Furthermore, Shahin et al. ${ }^{29}$ investigated the influence of RVE with different particle positions (randomness) on the FEM $\times$ DEM multiscale modelling. Liu et al. ${ }^{30}$ developed a nonlocal multiscale discrete-continuum model based on the generalised mathematical homogenisation scheme. Argilaga et al. ${ }^{31}$ enhanced the coupled technique by adopting operators different from the consistent tangent matrix and by developing ad-hoc solution strategies to improve the stability and numerical efficiency of the method. Liu et al. ${ }^{32}$ combined the hierarchical FEM-DEM approach with a servo-control methodology to improve the micro-scale boundary conditions.

Although the multiscale analysis using DEM-FEM provides new insights into some complex geotechnical problems, it is still challenging to simulate granular materials with large deformation due to mesh distortion issues in FEM ${ }^{33,34}$. To overcome this difficulty, researchers attempted to combine DEM and MPM as an alternative to the FEM-DEM technique. Compared to FEM, MPM is more suitable for modelling problems involving large deformation because the complicated remeshing is avoided ${ }^{34}$. Liu et al. ${ }^{3,35}$ first developed a coupled MPM-DEM technique and applied it to simulate granular flows that impact solid blocks. Then, Liang and Zhao ${ }^{36}$ coupled DEM with MPM to investigate large deformation of granular materials in geomechanics.

Currently, applications of multiscale techniques to the simulation of granular geomaterials are still uncommon due to high demanding computing costs. This trend will change with the development of emerging heterogeneous computer architectures, 
such as CPU and GPU clusters. In such a multiscale technique, RVE is the key element because its computational efficiency and accuracy play a dominant role in the multiscale analysis. In existing multiscale techniques where RVE is used to replace constitutive models, homogeneous deformation tends to be assumed in the RVE, which is, however, inadequate for problems involving large deformation. Another issue with existing methods is that the RVE box can become severely distorted in large deformation cases. When an RVE box becomes slender in any direction it will lose the representative nature and thus cannot be regarded as a valid RVE anymore. The contact detection between boundary particles and those image particles is also an issue that can be conducted more efficiently. In the conventional deformed RVE model, the number of boundary particles can increase gradually under a consecutive shearing deformation. Hence more computational cost and memory are required for handling the interaction of boundary particles and those image particles from the opposite side.

Given the aforementioned problems, this work aims to improve existing granular RVE models by adapting a recently proposed RVE model ${ }^{37}$ for molecular dynamics (MD). To overcome the distortion of the RVE box and the associated time-consuming contact detection between boundary particles and image particles, an adaptive RVE model equipped with an evolutionary periodic boundary (EPB) algorithm is developed, by which the mentioned issues can be well resolved.

\section{An adaptive granular RVE model}

An RVE is the smallest volume over which a measurement can be made to yield a representative value for the whole system. It is often used in studying material properties and multiscale simulations. Fig. 1 illustrates the RVE model used in existing multiscale methods, where the RVE box deforms with loading.

Different from most multiscale methods where homogeneous deformation is assumed within the RVE model, we adopt an RVE model that is proposed for a coupled MDMPM technique ${ }^{37}$, in which the strain rate or velocity gradient is assumed the same. 
This assumption is general and could be more reasonable in problems involving large deformation. The velocity gradient $\nabla v$ passed to the RVE model from the macroscopic method, MPM, can be uniquely decomposed into an upper triangular matrix of strain rate and a spin tensor as

$$
\nabla \boldsymbol{v}=\left[\begin{array}{lll}
\frac{\partial v_{x}}{\partial x} & \frac{\partial v_{x}}{\partial y} & \frac{\partial v_{x}}{\partial z} \\
\frac{\partial v_{y}}{\partial x} & \frac{\partial v_{y}}{\partial y} & \frac{\partial v_{y}}{\partial z} \\
\frac{\partial v_{z}}{\partial x} & \frac{\partial v_{z}}{\partial y} & \frac{\partial v_{z}}{\partial z}
\end{array}\right]=\left[\begin{array}{ccc}
\dot{\varepsilon}_{x x} & 2 \dot{\varepsilon}_{x y} & 2 \dot{\varepsilon}_{x z} \\
0 & \dot{\varepsilon}_{y y} & 2 \dot{\varepsilon}_{y z} \\
0 & 0 & \dot{\varepsilon}_{z z}
\end{array}\right]+\left[\begin{array}{ccc}
0 & -w_{z} & w_{y} \\
w_{z} & 0 & -w_{x} \\
-w_{y} & w_{x} & 0
\end{array}\right]
$$

where $\dot{\varepsilon}_{i j}$ is the strain rate components with $i, j=x, y, z$. It should be highlighted that the decomposition (Eq. 1) is different from the standard decomposition in continuum mechanics, where the velocity gradient is decomposed into a symmetric strain rate tensor and an anti-symmetric spin tensor associated with the vorticity. The uppertriangular strain rate matrix plays a key role in the determination of image particles below (see Eqs. 6-9), and therefore will be further explained in our evolutionary periodic boundary algorithm to be presented in Table 1 .

In the study of constitutive models, the principle of objectivity indicates that the rotation transformation of coordinates does not affect the stress. Therefore, for the large deformation problem with a low strain rate, the spin tensor can be neglected. To minimise the effect of RVE boundaries, the periodic boundary is always applied in RVE simulations. However, the deformed RVE algorithm in existing multiscale methods is only valid for problems with limited or moderate deformation. When subjected to large deformation, the RVE box may become a slender diamond or parallelogram in twodimensional cases as shown in Fig. 2, thereby losing its representative nature due to the presence of only limited particles in the narrow direction. In three-dimensional (3D) cases, severe distortion of an RVE box can also be encountered. In such a situation, the reinitialization of the RVE box becomes essential.

To overcome the aforementioned problems, an advanced RVE model that can account for the deformation history of the deformed RVE domain was recently proposed ${ }^{37}$ by 
using a lattice matrix $\boldsymbol{L}(t) . \boldsymbol{L}(t)$ records the deformation history of a deformed RVE box, and takes the form

$$
\boldsymbol{L}(t)=\left[\begin{array}{lll}
L_{x x} & L_{x y} & L_{x z} \\
L_{y x} & L_{y y} & L_{y z} \\
L_{z x} & L_{z y} & L_{z z}
\end{array}\right]
$$

where $L_{x x}, L_{y y}, L_{z z}$ are the axial lengths (projection lengths along coordinate axes) of the deformed RVE domain, respectively; and the off-diagonal elements represent the shearing deformation of the RVE in different directions. Initially, the off-diagonal elements, $L_{i j}(i \neq j)$, of a cubic RVE domain are set to be zero.

In this advanced RVE model, the RVE box always maintains a cuboid shape. Consider a 2-dimensional (2D) case subjected to a shear deformation as shown in Fig. 3 where the initial lattice (i.e. the RVE domain) at time $t_{1}$ is shown on the left. A timestep later the lattice becomes a parallelogram as shown by the dotted lines on the right due to the deformation. The lattice dimensions increase from $L_{x x}\left(t_{1}\right)$ and $L_{y y}\left(t_{1}\right)$ to $L_{x x}\left(t_{2}\right)$ and $L_{y y}\left(t_{2}\right)$ respectively and the shearing deformation increases from zero to $L_{x y}\left(t_{2}\right)$. To avoid the reinitialization of the deformed RVE that has undergone large deformation, a new rectangle in bold, with the same axial lengths $\left(L_{x x}\left(t_{2}\right)\right.$ and $\left.L_{y y}\left(t_{2}\right)\right)$ as the deformed RVE box, is introduced to represent material properties around a material point or Gauss point in continuum models. Following a similar procedure, a deformed 3D RVE can be replaced by a cuboid RVE. This new rectangle (2D) or cuboid (3D), with the Gauss or material point as its origin, will be the new RVE domain for the next time step. Depending on the specific deformation state, the dimension of the new RVE may change over time. Here we coin the term "adaptive RVE" to denote this continuously updated rectangle or cuboid, as it is adjusted every single timestep according to the periodicity of the deformed RVE model.

Then, the particles outside this new adaptive RVE need to be mapped back to the domain using their image counterparts. Let $\boldsymbol{x}_{\boldsymbol{o}}(t)$ be the position of a particle outside the domain at time $t$, its image counterpart within the domain is given by $\boldsymbol{x}_{\boldsymbol{i}}(t, \boldsymbol{n})$

$$
\boldsymbol{x}_{\boldsymbol{o}}(t)=\left\{x_{o}, y_{o}, z_{o}\right\}
$$




$$
\boldsymbol{x}_{\boldsymbol{i}}(t, \boldsymbol{n})=\boldsymbol{x}_{\boldsymbol{o}}(t)+\boldsymbol{L}(t) \cdot \boldsymbol{n}
$$

where $\boldsymbol{L}(t)$ is the lattice matrix of the deformed RVE; $\boldsymbol{n}$ is a column vector of the periodicity numbers in the three axial directions and given by

$$
\boldsymbol{n}=\left\{n_{x}, n_{y}, n_{z}\right\}^{T}
$$

The deformation history of the lattice matrix of an RVE can be updated by the deformation gradient $\boldsymbol{F}$ at the macroscopic level

$$
\boldsymbol{L}(t+\Delta t)=\boldsymbol{F} \cdot \boldsymbol{L}(t)=(I+\nabla \boldsymbol{v} \Delta t) \cdot \boldsymbol{L}(t)=\left(I+\left[\begin{array}{ccc}
\dot{\varepsilon}_{x x} & 2 \dot{\varepsilon}_{x y} & 2 \dot{\varepsilon}_{x z} \\
0 & \dot{\varepsilon}_{y y} & 2 \dot{\varepsilon}_{y z} \\
0 & 0 & \dot{\varepsilon}_{z z}
\end{array}\right] \Delta t\right) \cdot \boldsymbol{L}(t)
$$

As the lattice matrix $\boldsymbol{L}(t)$ is updated from the upper-triangular strain rate matrix, the lattice matrix is also upper-triangular. Substituting Eq. 6 into Eq. 4 gives the mapped coordinates of the image particle

$$
\begin{gathered}
x_{i}(t, \boldsymbol{n})=x_{o}(t)+L_{x x} n_{x}+L_{x y} n_{y}+L_{x z} n_{z} \\
y_{i}(t, \boldsymbol{n})=y_{o}(t)+L_{y y} n_{y}+L_{y z} n_{z} \\
z_{i}(t, \boldsymbol{n})=z_{o}(t)+L_{z z} n_{z}
\end{gathered}
$$

Assuming that the velocity gradient around a material point or Gauss point is constant, the velocity distribution of particles inside the RVE box is linear. Therefore, the velocity of an image particle in the new RVE box can be updated based on the distance between its original position and the current position by

$$
\boldsymbol{v}_{i}(t)=\boldsymbol{v}_{o}(t)+\nabla \boldsymbol{v} \cdot(\boldsymbol{L}(t) \cdot \boldsymbol{n})
$$

where $\boldsymbol{v}_{o}$ is the velocity of the original particle, $\boldsymbol{v}_{i}$ is the velocity of the image particle in the new RVE box and its component form is given as follows

$$
\begin{gathered}
v_{i x}=v_{o x}+\dot{\varepsilon}_{x x}\left(x_{i}-x_{o}\right)+2 \dot{\varepsilon}_{x y}\left(y_{i}-y_{o}\right)+2 \dot{\varepsilon}_{x z}\left(z_{i}-z_{o}\right) \\
v_{i y}=v_{o y}+\dot{\varepsilon}_{y y}\left(y_{i}-y_{o}\right)+2 \dot{\varepsilon}_{y z}\left(z_{i}-z_{o}\right) \\
v_{i z}=v_{o z}+\dot{\varepsilon}_{z z}\left(z_{i}-z_{o}\right)
\end{gathered}
$$


The theoretical part of the adaptive RVE model for granular materials has been addressed above. The modelling of such an RVE model is mainly using DEM in a multiscale technique. Within each time step, the acceleration and velocity of a particle are assumed constant, based on the assumption that if a sufficiently small timestep is taken, the stress wave arising at the contact point of two particles will not propagate further to other particles. Hence, the contact interaction is only local. In DEM, the time integration of particle motion is achieved by the central difference method. The movement of a particle is governed by Newton's second law

$$
\begin{gathered}
m a+c \boldsymbol{v}=\boldsymbol{f}_{\boldsymbol{c}}+\boldsymbol{b} \\
I \ddot{\boldsymbol{\theta}}=\boldsymbol{T}_{\boldsymbol{c}}
\end{gathered}
$$

where $m$ is the mass of the particle; $\boldsymbol{a}$ and $\boldsymbol{v}$ are the acceleration and velocity, respectively; $c$ is the damping coefficient; $\boldsymbol{f}_{\boldsymbol{c}}$ is the resultant contact force acting on the particle; $\boldsymbol{b}$ is the body force; $I$ is the second moment of inertia of the particle; $\boldsymbol{\theta}$ is the rotation angle; and $\boldsymbol{T}_{\boldsymbol{c}}$ is the torque due to the tangential force applied at the contact point.

The contact force model used to handle the particle-particle collision can be treated as a serial connection of a dashpot and a spring. The spring has normal and tangential components. For spherical particles, the normal force $\boldsymbol{f}_{n}$ can be modelled by the Hertz contact model given by Eq. 16 and the tangential part $\boldsymbol{f}_{t}$ can be accounted for by the Coulomb friction model given by Eq. 17.

$$
\begin{gathered}
\boldsymbol{f}_{\boldsymbol{n}}=k_{n} \boldsymbol{\delta}_{n}^{3 / 2} \\
\boldsymbol{f}_{t}=\min \left\{\boldsymbol{f}_{t}^{p}+k_{t} \Delta \boldsymbol{\delta}_{t}, \mu \boldsymbol{f}_{n}\right\}
\end{gathered}
$$

where $k_{n}$ and $k_{t}$ are the normal and tangential stiffnesses of contact; $\boldsymbol{\delta}_{n}$ is the relative normal displacement; $\boldsymbol{f}_{t}^{p}$ is the tangential force at the previous time step, and $\Delta \boldsymbol{\delta}_{t}$ is the incremental tangential displacement between two particles; $\mu$ is the coefficient of friction. The dashpot with a constant damping coefficient $c$ is used to characterise energy dissipation during collision. 


\section{Macroscopic Cauchy stress and evolutionary periodic boundary}

In the hierarchical multiscale modelling approach, the macroscopic method (FEM or MPM) is used to calculate the overall deformation of granular materials at the engineering scale, while the microscopic method (DEM) is utilised to compute the stress in the RVE model at the grain level. At each time step, the deformation gradient calculated by FEM or MPM is passed to DEM, from which the microscopic models will compute the averaged Cauchy stress of the RVE based on the induced particle geometry and contact forces. Then, the averaged stress will be used in the macroscopic simulation at the next time step.

In a granular particle system, if the macroscopic length scale is sufficiently large compared to the characteristic particle size, the generalized macroscopic momentum equation ${ }^{38,39}$ can be written as

$$
\frac{\partial \rho \widetilde{\boldsymbol{v}}}{\partial t}+\nabla \cdot(\rho \widetilde{\boldsymbol{v}})=\nabla \cdot \boldsymbol{\sigma}+\rho \boldsymbol{b}
$$

where $\rho$ is the bulk density of a granular packing; $\widetilde{\boldsymbol{v}}$ is the averaged velocity of the material point in continuum-based models; and $\boldsymbol{\sigma}$ is the Cauchy stress defined in continuum mechanics.

The Cauchy stress can be expressed as the combination of the Reynolds stress $\sigma_{R}$ and the contact stress $\boldsymbol{\sigma}_{\boldsymbol{c}}$

$$
\sigma=\sigma_{R}+\sigma_{c}
$$

where the Reynolds stress $\boldsymbol{\sigma}_{\boldsymbol{R}}$ is defined by the velocity fluctuation $\left(\boldsymbol{v}_{i}-\widetilde{\boldsymbol{v}}\right)$ of each particle:

$$
\boldsymbol{\sigma}_{\boldsymbol{R}}=-\frac{1}{V} \sum_{i=1}^{n} m_{i}\left(\boldsymbol{v}_{i}-\widetilde{\boldsymbol{v}}\right)\left(\boldsymbol{v}_{i}-\widetilde{\boldsymbol{v}}\right)
$$

where $V$ is the volume of the particle packing; $n$ is the total number of particles; $m_{i}$ and $\boldsymbol{v}_{i}$ are, respectively, the mass and velocity of the particle $i$. The contact stress $\sigma_{R}$ is defined as 


$$
\boldsymbol{\sigma}_{\boldsymbol{c}}=\frac{1}{V} \sum_{i=1}^{n} \sum_{j=1}^{n_{c}^{i}} \boldsymbol{R}_{\boldsymbol{i}} \boldsymbol{f}_{\boldsymbol{i j}}
$$

where $n_{c}^{i}$ is the total contact number of particle $i$ with surrounding particles; $R_{i}$ is the radius of particle $i$ and $\boldsymbol{f}_{\boldsymbol{i j}}$ is the contact force between particles $i$ and $j$.

Both the Reynolds stress $\sigma_{R}$ and the contact stress $\sigma_{c}$ can be directly calculated from the RVE based microscopic simulations. It is reported that the Reynolds stress is at least one order smaller than the contact stress in a dense granular flow. This will also be demonstrated by our RVE simulations.

The Cauchy stress is calculated from the particles in the RVE including the boundary particles and is then used to launch the macroscopic computation in the multiscale modelling. However, for the contact stress, the contact between boundary particles and their images at the opposite face also need to be considered. In the conventional deformed RVE model, the number of boundary particles could increase gradually due to the continuous shearing deformation. Take a simple shear case similar to the one shown in Fig. 3 as an example. The growth of the inclined lengths (for 2D) or areas (for 3D) of the left and right boundaries will lead to an increase of the number of boundary particles, while the boundary lengths/areas of other boundary faces remain constant. Hence more computational costs are required for handling the interaction between boundary particles and their image particles at the opposite face with the evolution of shear deformation.

Next we will propose a general and efficient periodic boundary algorithm for detecting contacts of boundary particles. This algorithm is based on the above adaptive RVE model and considers the deformation history of the RVE box using the lattice matrix $\boldsymbol{L}(t)$. The basic idea of this algorithm is to divide the RVE domain/lattice into regular grids with each grid cell accommodating 10 to 20 particles and find the images of the particles at the boundary layers. Eqs. 7-9 indicate that the $x$ coordinate of a particle is related to its movement in the $x, y$ and $z$ directions, while the shift of the particle along the $x$-direction does not affect its $y$ and $z$ coordinates. Therefore, for a shear deformation along the $x$-direction, it is more effective to process the image particles at the top and 
bottom boundaries first and then to the image particles from the left and right boundaries including the images of the bottom and top boundary particles. This step is the key to avoiding the complicated treatment of corner particles. A full EPB algorithm for $3 \mathrm{D}$ cases is summarised in Table 1 below:

Table 1 The 3D evolutionary periodic boundary (EPB) algorithm

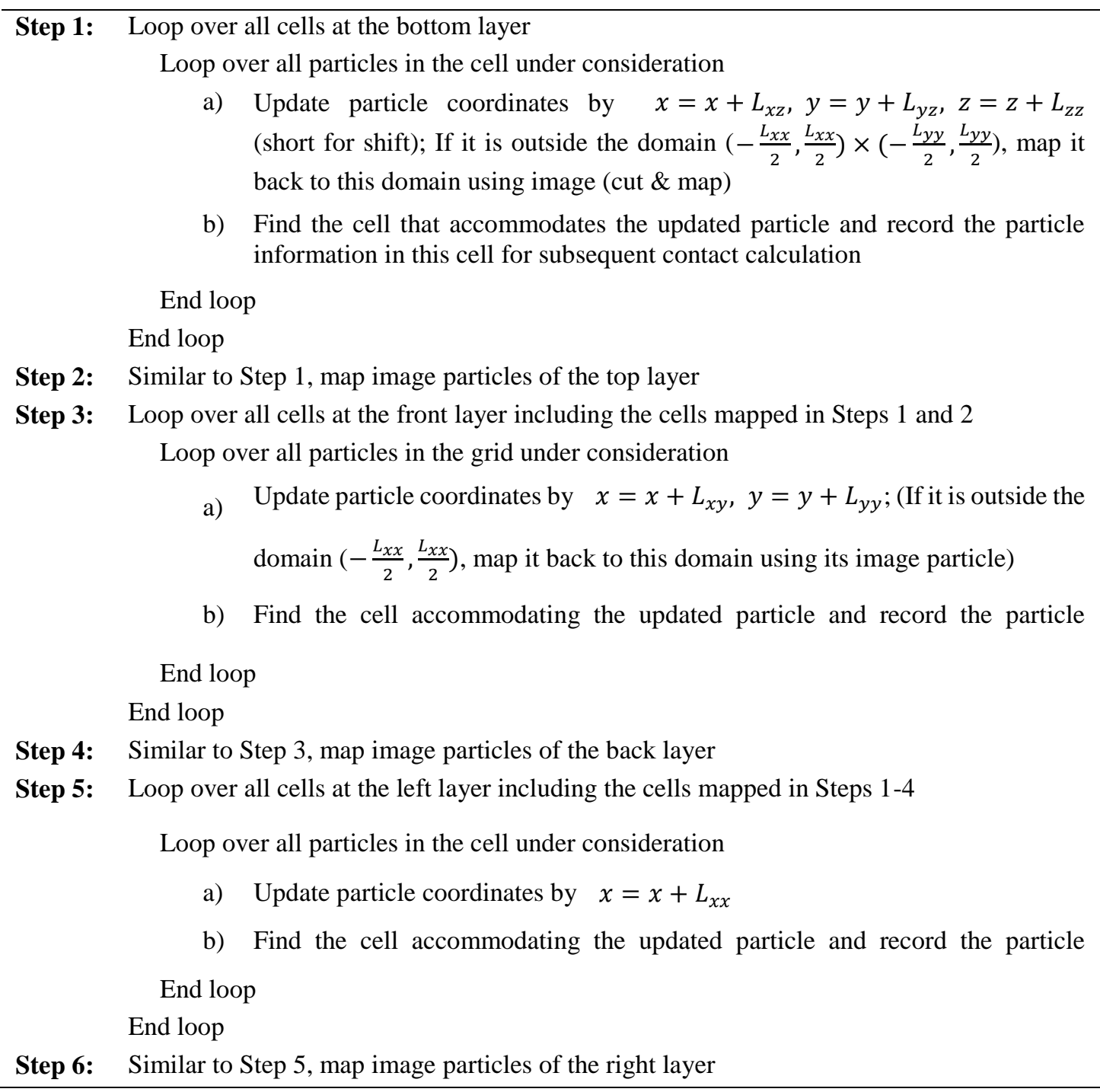

To facilitate the understanding of the proposed EPB algorithm, a two-dimensional procedure is illustrated in Fig. 4, where the primary steps are included.

In our proposed evolutionary periodic boundary, the total number of boundary particles are almost unchanged due to the constant total boundary area in a simple shear problem. 
In contrast to the conventional deformed RVE box, fewer boundary particles and images need to be handled.

\section{Numerical examples}

\subsection{Simple shear and complex-loading tests}

To validate the proposed adaptive RVE model with the EPB algorithm, we consider a 3D system of 864 particles undergoing an extremely large simple shear deformation. For simplicity, we only adopt spherical particles of uniform size in this work. The granular model with porosity $(n=0.548)$ is shown in Fig. 5. The dimensions $L_{x x}, \quad L_{y y}, \quad L_{z z}$ of this RVE box are all $2.0 \mathrm{~m}$; the particle diameter is $0.2 \mathrm{~m}$ and the density is $2650 \mathrm{~kg} / \mathrm{m}^{3}$. This granular model can be treated as an initial representative element of a material point in the macroscopic simulations of a debris flow. The normal and tangential stiffnesses of contact are $2 \times 10^{7} \mathrm{~N} / \mathrm{m}$ and $2 \times 10^{6} \mathrm{~N} / \mathrm{m}$, respectively. The coefficient of friction used in the Coulomb friction model is 0.3. The damping coefficient $c$ is $20 \mathrm{Ns} / \mathrm{m}$. The timestep is $3.688 \times 10^{-5} \mathrm{~s}$. The total simulated physical time is $20 \mathrm{~s}$. Initially, particles do not interact with each other and are stationary. To mimic a simple shear deformation, a shear rate $\left(\dot{\gamma}_{x z}=2 \dot{\varepsilon}_{x z}=0.5\right)$ is applied to the RVE model, which gives a total shear deformation of $10 L_{x x}$. Because of the applied shear rate $\dot{\gamma}_{x z}$, the particle velocity $\left(v_{x}\right)$ is proportional to its $z$ coordinate (i.e. $\frac{\partial v_{x}}{\partial z}=\dot{\gamma}_{x z}$ ). This relatively simple system has been studied extensively, mainly as a means of assessing the validity of kinetic theories.

The configurations of the particles and their velocities $v_{x}$ at four different shear deformation stages are given in Fig. 6. During the simple shearing, the upper and bottom boundaries remain parallel and maintain a constant distance $\left(L_{z z}\right)$, but are translated relative to each other. By selecting the origin of the coordinate system at the centre of the RVE box, it can be found that the lateral movements of particles within the upper and lower domain are exactly opposite against the $x-y$ plane. Although 
particles collide with their neighbours, their velocities $\left(v_{x}\right)$ are generally proportional to their $z$ coordinates at the end of each time step.

It should be noted that the total shear deformation $\left(L_{x z}=10 L_{x x}\right)$ of this RVE model is extremely large. For such a case, the conventional deformed RVE domain will deform into a slender parallelepiped as shown in Fig. 7. Within a conventional hierarchical multiscale modelling framework, such a distorted RVE box may give rise to three issues in a standard RVE model:

1) the increasing large deformation may deteriorate the representative capability of a traditional RVE in the narrow direction along which it is insufficient to apply the periodic boundary on the RVE domain;

2) for a three-dimensional case, an RVE box could be distorted/twisted under complex loading conditions, giving rise to a great challenge in tracking the RVE boundaries;

3) it may be unreasonable to assume that the whole deformed RVE undergoes uniform strain condition in such a slender domain of the deformed RVE.

However, as demonstrated by the current example, these issues have been well resolved by our proposed periodic boundary. No matter how large a shear deformation occurs, the adaptive RVE box is always maintained as a cube in this simulation.

To quantitatively characterise the mechanical behaviour of the RVE model undergoing large deformation, variations of the diagonal and off-diagonal components of the contact stress are given in Fig. 8. It is found that the diagonal components ( $\sigma_{x x}$ and $\left.\sigma_{z z}\right)$ increase dramatically when the loading is applied, and then gradually decrease to some constant values. This phenomenon is associated with the evolution of angular velocities of particles and has been reported in granular physics ${ }^{39,40}$. Particles are at rest at the beginning, but once the shear rate $\left(\dot{\gamma}_{x z}\right)$ is applied, the angular velocity of particles increases quickly due to interactions with neighbours. It is also found that the stress components $\sigma_{x x}$ and $\sigma_{z z}$ are always greater than $\sigma_{y y}$, because the shear loading is applied along the $x$ direction with the gradient in the $z$ direction. From Fig.8b, we can see that the off-diagonal components of the contact stress are symmetric and lower 
than the diagonal components. Compared to the $\sigma_{x z}$ and $\sigma_{z x}$ components, other offdiagonal components can be ignored.

Fig. 9 shows the variation of the primary components of the Reynolds stress which is the other part of the Cauchy stress. Similar to the contact stress, the magnitudes of $\sigma_{x x}$ and $\sigma_{z z}$ of the Reynolds stress are smaller than $\sigma_{y y}$. Particularly, the Reynolds stress is at least one order smaller than the contact stress in the current granular model (porosity $n=0.548$ ).

The above simple shear problem, where only shearing deformation is considered and the adaptive RVE domain is constant, clearly demonstrates the merits of the adapted RVE model with an evolutionary periodic boundary for problems involving large deformation.

To further illustrate the adaptive RVE model, we consider a more complex loading case with $2 \dot{\varepsilon}_{x z}=1.5$ and $\dot{\varepsilon}_{x x}=-0.03$ so that the adaptive RVE domain is changing. The granular packing is comprised of 463 particles with particle diameter ranging from 0.19 to $0.28 \mathrm{~m}$. Initially, the dimensions $L_{x x}, L_{y y}, L_{z z}$ of this RVE box are all $2.0 \mathrm{~m}$. The material parameters are the same as the previous simple shear case. Snapshots of the RVE model and variations of particle velocity at different instants are shown in Fig. 10. Similar to the simple shear case, the velocity distribution is spatially linear due to the applied shear rate. In particular, the RVE domain is reducing in the $x$ direction with time. The specific evolution of the diagonal components of the lattice matrix is shown in Fig. 11.

\subsection{Effects of porosity on the mechanical behaviour of granular materials}

Next, we will investigate the effect of the solid volume fraction or porosity on the mechanical response of granular flows subject to large deformation using the proposed algorithm. To achieve this goal, two more RVE models with porosities of 0.738 and 0.282 respectively are generated (see Fig. 12). These two RVE models, together with the above model with $n=0.548$, will be used as representatives for loose, medium 
dense and dense specimens, respectively. These RVE models share the same dimensions and particle-scale properties and undergo the same external shear rates.

Fig. 13 compares the variations of the hydrostatic pressure $\left(p=-\frac{\sigma_{i i}}{3}\right)$ of the three RVE models. Apparently, the pressure increases with the decrease of the porosity. Similar results can be found in the off-diagonal components. For simplicity, only the component $\sigma_{x z}$ is compared in Fig. 14 .

Shear localisation is a remarkable sign of the instability of ductile solid materials, such as granular materials. When the material deforms sufficiently into the plastic state, a homogeneous deformation pattern gives way to highly localised deformation featured as a "shear band" 41 . It mainly occurs in dense granular materials under large deformations. The conventional RVE model with homogeneous deformation assumption is unable to reasonably reflect such a strain localisation phenomenon at the RVE level. To overcome this issue, attempts have been made to improve the conventional RVE model by introducing high-order continuum mechanics, such as Corsserat continuum ${ }^{42,43}$ and micromorphic continuum ${ }^{44,45}$ to RVE. If the RVE domain is much smaller than the characteristic length of macroscopic shear bands, the RVE is still a representative of the local material point. It is found that our adaptive RVE model based on the classical continuum mechanics is capable of capturing shear localisation. The shear localisation phenomenon can be reflected from the strainsoftening effect of the stress-strain curves, which is shown in Fig.14.

To examine the relationship between the Reynolds stress $\left(\boldsymbol{\sigma}_{\boldsymbol{R}}\right)$ and the contact stress $\left(\boldsymbol{\sigma}_{\boldsymbol{c}}\right)$, four more test cases with different porosities are further carried out. Variations of the ratio of the trace of the Reynolds stress $\left(\sigma_{i i_{-}} R\right)$ to the trace of the contact stress $\left(\sigma_{i i_{-}} c\right)$ are given in Fig.15, which shows that the ratio increases with the increase of the porosity. For the two dense packings ( $n=0.282$ and $n=0.371)$, the Reynolds stress is at least three orders smaller than the contact stress; while in the loose packing ( $n=$ 0.738), they are of a similar scale: the Reynolds stress is only half of the contact stress. This indicates that the Reynolds stress in a loose granular system is as important as the 
contact stress; while for dense particle packings, the Reynolds stress can be ignored. This conclusion is consistent with the findings in the field of granular physics for a dense granular system. The above results also confirm that it is acceptable to only consider the contact stress as Cauchy stress in multiscale simulations for most geotechnical problems where medium dense or dense granular specimen are mainly encountered.

\section{Concluding remarks}

This study has proposed an enhanced granular RVE model aiming to extend the applicability of multiscale techniques to problems encountering extremely large deformation. The key contribution is the proposed adaptive RVE box and the associated evolutionary periodic boundary algorithm. Meanwhile, to account for the deformation history of the RVE lattice, the lattice matrix $\boldsymbol{L}$ is used to effectively map the images of boundary particles. The simple shear test of the three granular packings with various porosities have illustrated the applicability and capability of the proposed RVE model. The results also show that for a granular model subjected to a large deformation process, Reynolds stress can be ignored for dense particle packings; while for loose particles systems, Reynolds stress and contact stress are of the same importance. The essential features of the proposed adaptive RVE model can be summarised as follows:

(1) In the adaptive RVE model, the gradient of deformation, instead of homogenous deformation in existing RVE models, is achieved by using a constant velocity gradient, and the velocity gradient is decomposed into an upper-triangular strain rate matrix and a spin tensor. The latter can be ignored for problems involving only a small strain rate.

(2) This adaptive RVE model is mass conserved. During the whole simulation, only the original inhabitant particles or their image particles will be included in the new adaptive RVE box, and no new particles will be generated or removed. Therefore, the number of particles in the simulation always remains the same. 
(3) By only considering the first order gradient of deformation, the adaptive RVE model is capable of capturing shear localisation at the RVE level in the framework of classical continuum mechanics.

(4) Compared to the conventional deformed RVE model, our evolutionary periodic boundary algorithm can treat the interaction between boundary particles and image particles at the opposite faces more efficiently.

It should be noted that the effect of the spin tensor is ignored for small strain rate problems in this work. Its effects on high strain rate problems need further investigation. In addition, the present adaptive RVE model is limited in overcoming the invalidity of a slender RVE box caused by extremely large compression deformation. Improvements of this adaptive model to incorporate spin tensor and to resolve the large compression deformation problems will be reported elsewhere.

\section{References}

1. Rojek J, Oñate E. Multiscale analysis using a coupled discrete/finite element model. Interaction and Multiscale Mechanics 2007;1(1):1-31.

2. Wellmann C, Wriggers P. A two-scale model of granular materials. Computer Methods in Applied Mechanics and Engineering 2012;205-208:46-58.

3. Liu C, Sun Q, Zhou GG. Coupling of material point method and discrete element method for granular flows impacting simulations. International Journal for Numerical Methods in Engineering 2018;115(2):172-188.

4. Qu T, Wang S, Hu Q. Coupled discrete element-finite difference method for analysing effects of cohesionless soil conditioning on tunneling behaviour of EPB shield. KSCE Journal of Civil Engineering 2019;23(10):4538-4552.

5. Liu WK, Karpov EG, Park HS. Nano mechanics and materials: theory, multiscale methods and applications. John Wiley \& Sons; 2006.

6. Tu X, Andrade JE, Chen Q. Return mapping for nonsmooth and multiscale elastoplasticity. Computer Methods in Applied Mechanics and Engineering 2009;198(30-32):2286-2296.

7. Qu T, Wang S, Fu J, Hu Q, Zhang X. Numerical examination of EPB shield tunneling-induced responses at various discharge ratios. Journal of Performance of Constructed Facilities 2019;33(3):04019035.

8. Kouznetsova V, Brekelmans W, Baaijens F. An approach to micro-macro modeling of heterogeneous materials. Computational mechanics 2001;27(1):37-48. 
9. Terada K, Kikuchi N. A class of general algorithms for multi-scale analyses of heterogeneous media. Computer methods in applied mechanics and engineering 2001;190(40-41):5427-5464.

10. Kouznetsova V, Geers MG, Brekelmans WM. Multi-scale constitutive modelling of heterogeneous materials with a gradient-enhanced computational homogenization scheme. International journal for numerical methods in engineering 2002;54(8):1235-1260.

11. Kaneko K, Terada K, Kyoya T, Kishino Y. Global-local analysis of granular media in quasi-static equilibrium. International Journal of Solids and Structures 2003;40(15):4043-4069.

12. Miehe C, Dettmar J. A framework for micro-macro transitions in periodic particle aggregates of granular materials. Computer Methods in Applied Mechanics and Engineering 2004;193(3-5):225-256.

13. Miehe C, Dettmar J, Zäh D. Homogenization and two-scale simulations of granular materials for different microstructural constraints. International Journal for Numerical Methods in Engineering 2010;83(8-9):1206-1236.

14. Andrade JE, Tu X. Multiscale framework for behavior prediction in granular media. Mechanics of Materials 2009;41(6):652-669.

15. Andrade JE, Avila C, Hall S, Lenoir N, Viggiani G. Multiscale modeling and characterization of granular matter: from grain kinematics to continuum mechanics. Journal of the Mechanics and Physics of Solids 2011;59(2):237-250.

16. Nitka M, Combe G, Dascalu C, Desrues J. Two-scale modeling of granular materials: a DEM-FEM approach. Granular Matter 2011;13(3):277-281.

17. Nitka M, Tejchman J. A Two-Scale Numerical Approach to Granular Systems/Wybrane Problemy Szacowania Prawdopodobienstwa Zawodu W Sytuacji Pozaru. Archives of Civil Engineering 2011;57(3):313-330.

18. Nguyen TK, Combe G, Caillerie D, Desrues J. FEM $\times$ DEM modelling of cohesive granular materials: numerical homogenisation and multi-scale simulations. Acta Geophysica 2014;62(5):1109-1126.

19. Guo N, Zhao J. A coupled FEM/DEM approach for hierarchical multiscale modelling of granular media. International Journal for Numerical Methods in Engineering 2014;99(11):789-818.

20. Guo N, Zhao J. Multiscale insights into classical geomechanics problems. International Journal for Numerical and Analytical Methods in Geomechanics 2016;40(3):367-390.

21. Guo N, Zhao J. 3D multiscale modeling of strain localization in granular media. Computers and Geotechnics 2016;80:360-372.

22. Guo N, Zhao J. Parallel hierarchical multiscale modelling of hydro-mechanical problems for saturated granular soils. Computer Methods in Applied Mechanics and Engineering 2016;305:37-61.

23. Guo N, Zhao J, Sun W. Multiscale analysis of shear failure of thick-walled hollow cylinder in dry sand. Géotechnique Letters 2016;6(1):77-82.

24. Lim K-W, Kawamoto R, Andò E, Viggiani G, Andrade JE. Multiscale characterization and modeling of granular materials through a computational 
mechanics avatar: a case study with experiment. Acta Geotechnica 2016;11(2):243253.

25. Wu H, Zhao J, Guo N. Multiscale insights into borehole instabilities in highporosity sandstones. Journal of Geophysical Research: Solid Earth 2018;123(5):3450-3473.

26. Xiong H, Yin ZY, Nicot F. A multiscale work-analysis approach for geotechnical structures. International Journal for Numerical and Analytical Methods in Geomechanics 2019;43(6):1230-1250.

27. Desrues J, Argilaga A, Caillerie D, et al. From discrete to continuum modelling of boundary value problems in geomechanics: An integrated FEM-DEM approach. International Journal for Numerical and Analytical Methods in Geomechanics 2019;43(5):919-955.

28. Zhao S, Zhao J, Lai Y. Multiscale modeling of thermo-mechanical responses of granular materials: A hierarchical continuum-discrete coupling approach. Computer Methods in Applied Mechanics and Engineering 2020;367:113100.

29. Shahin G, Desrues J, Pont SD, Combe G, Argilaga A. A study of the influence of REV variability in double-scale FEM $\times$ DEM analysis. International Journal for Numerical Methods in Engineering 2016;107(10):882-900.

30. Liu Y, Sun W, Yuan Z, Fish J. A nonlocal multiscale discrete-continuum model for predicting mechanical behavior of granular materials. International Journal for Numerical Methods in Engineering 2016;106(2):129-160.

31. Argilaga A, Desrues J, Dal Pont S, Combe G, Caillerie D. FEM $\times$ DEM multiscale modeling: Model performance enhancement from Newton strategy to element loop parallelization. International Journal for Numerical Methods in Engineering 2018;114(1):47-65.

32. Liu J, Bosco E, Suiker A. Multi-scale modelling of granular materials: numerical framework and study on micro-structural features. Computational Mechanics 2019;63(2):409-427.

33. Sulsky D, Zhou S-J, Schreyer HL. Application of a particle-in-cell method to solid mechanics. Computer physics communications 1995;87(1-2):236-252.

34. Zhang DZ, Ma X, Giguere PT. Material point method enhanced by modified gradient of shape function. Journal of Computational Physics 2011;230(16):63796398.

35. Liu C, Sun Q, Yang Y. Multi-scale modelling of granular pile collapse by using material point method and discrete element method. Procedia Engineering 2017;175:29-35.

36. Liang W, Zhao J. Multiscale modeling of large deformation in geomechanics. International Journal for Numerical and Analytical Methods in Geomechanics 2019;43(5):1080-1114.

37. Dhakal TR, Zhang DZ. Combining dual domain material point method with molecular dynamics for thermodynamic nonequilibriums. Journal of Computational Physics 2018;374:984-995.

38. Zhang D, Ma X, Rauenzahn R. Interspecies stress in momentum equations for dense binary particulate systems. Physical review letters 2006;97(4):048301. 
39. Ma X, Zhang DZ. Statistics of particle interactions in dense granular material under uniaxial compression. Journal of the Mechanics and Physics of Solids 2006;54(7):1426-1448.

40. Zhang DZ. Evolution of enduring contacts and stress relaxation in a dense granular medium. Physical Review E 2005;71(4):041303.

41. Qu T, Feng Y, Wang Y, Wang M. Discrete element modelling of flexible membrane boundaries for triaxial tests. Computers and Geotechnics 2019;115:103154.

42. Li X, Zhang S, Duan Q. A novel scheme for imposing periodic boundary conditions on RVE in second-order computational homogenization for granular material. Engineering Computations 2019;36(8):2835-2848.

43. Li X, Wang Z, Zhang S, Duan Q. Multiscale modeling and characterization of coupled damage-healing-plasticity for granular materials in concurrent computational homogenization approach. Computer Methods in Applied Mechanics and Engineering 2018;342:354-383.

44. D'Addetta G, Ramm E, Diebels S, Ehlers W. A particle center based homogenization strategy for granular assemblies. Engineering Computations 2004;21(2-4):360-383.

45. Biswas R, Shedbale A, Poh L. Nonlinear analyses with a micromorphic computational homogenization framework for composite materials. Computer Methods in Applied Mechanics and Engineering 2019;350:362-395. 


\section{Figures}

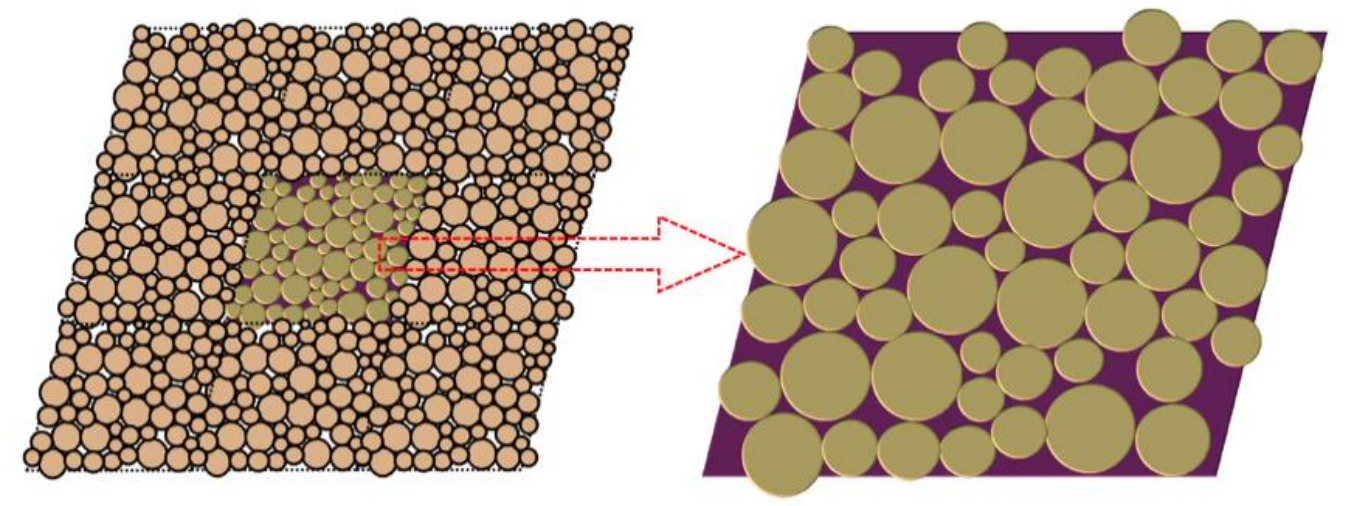

Fig. 1 Deformed RVE box under shearing in conventional multiscale analysis

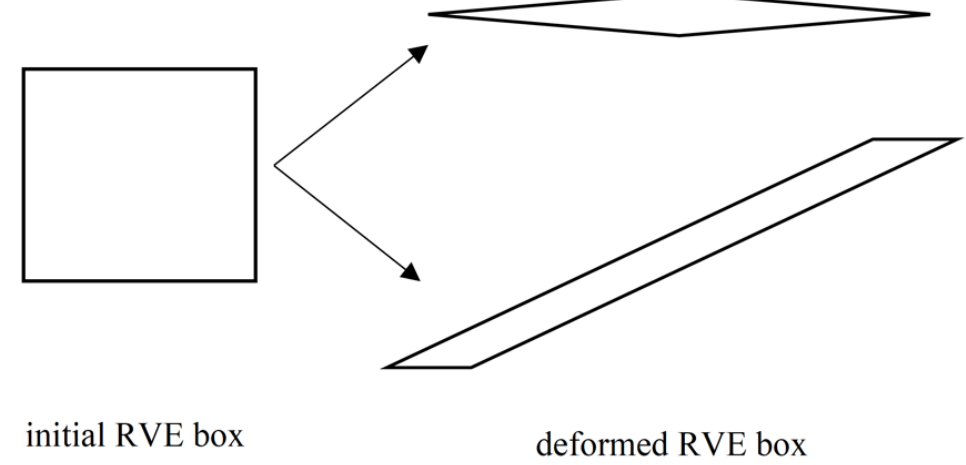

Fig. 2 Invalid RVE after large deformation

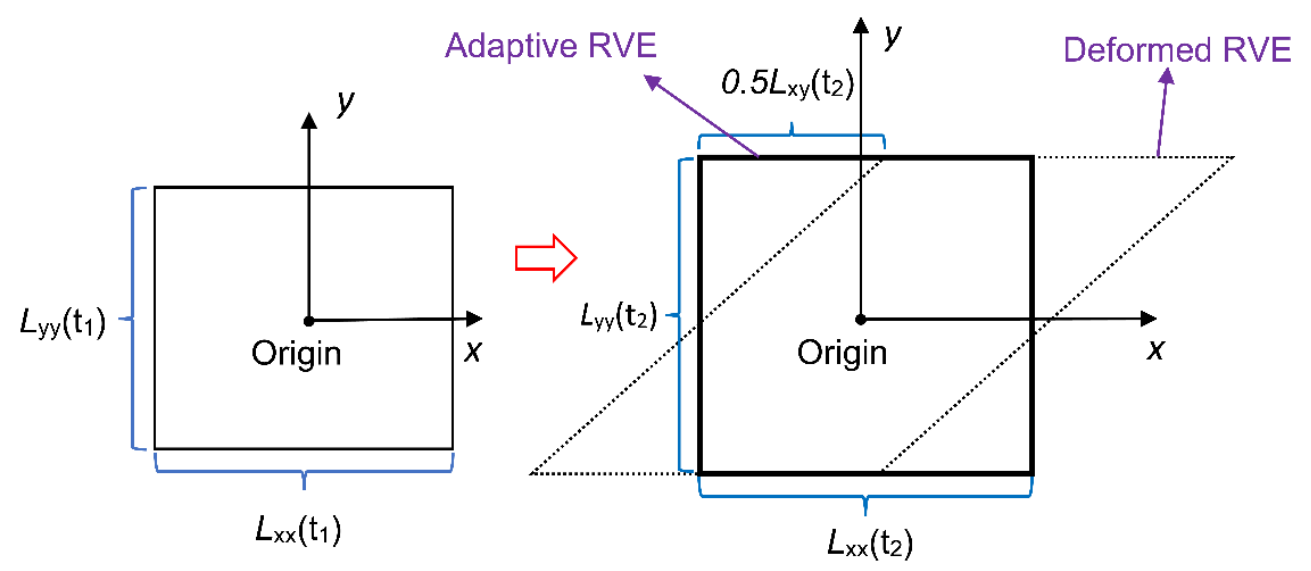

Fig. 3 The evolution of the adaptive RVE domain 


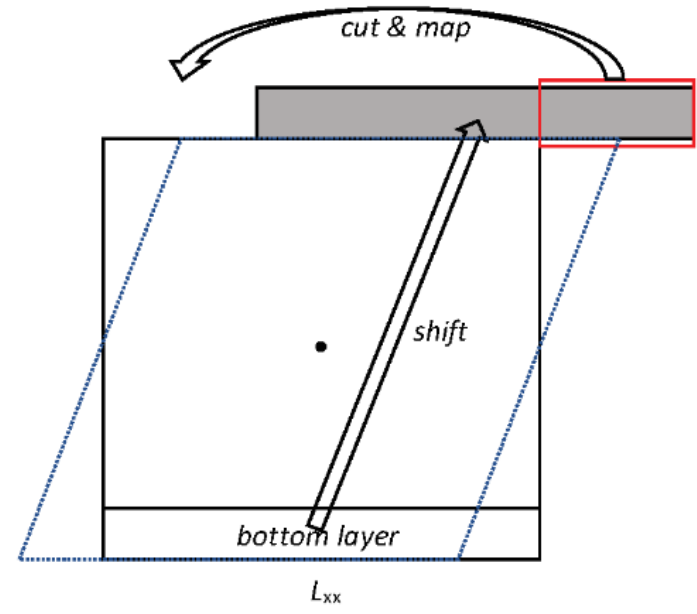

(a) Map image particles of the bottom layer

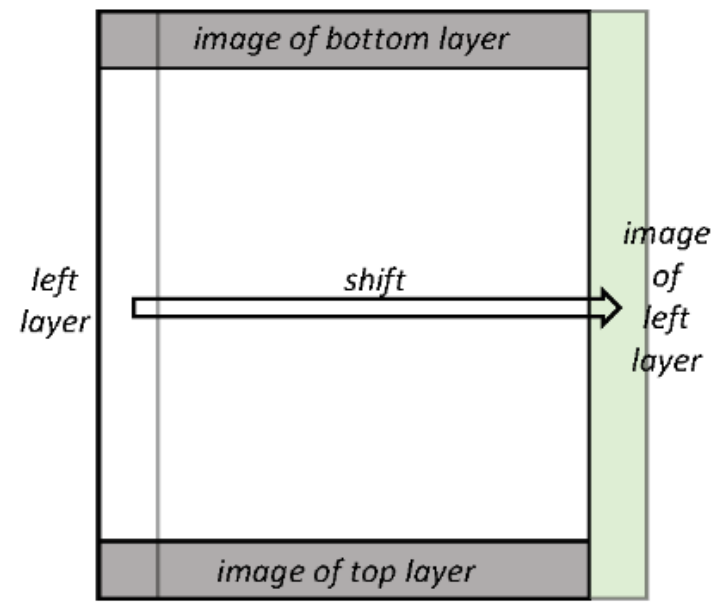

(b) Map image particles of the left layer

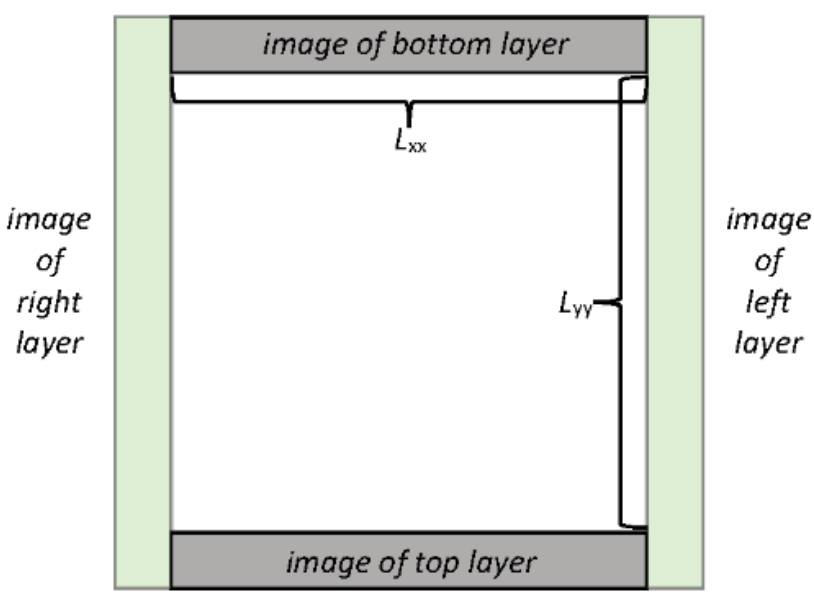

(c) Images of all boundary particles

Fig. 4 Schematic chart of 2D periodic boundary algorithm 


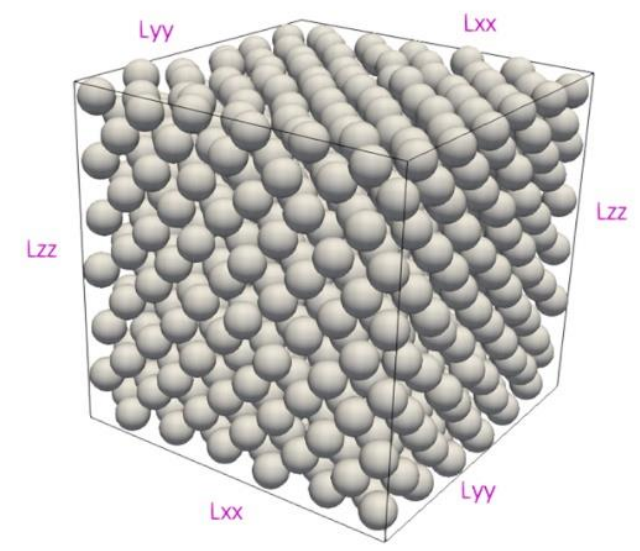

Fig. 5 Setup of granular RVE model $(n=0.548)$

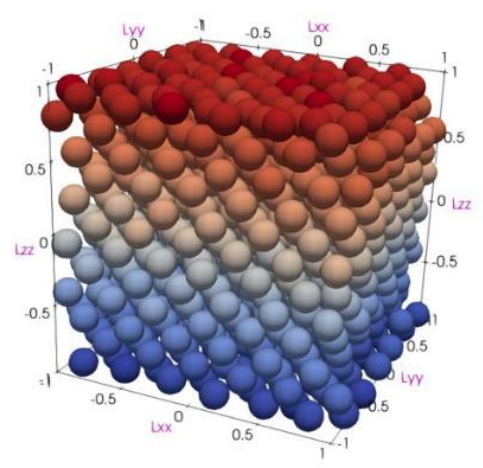

a) $\gamma_{x z}=0.1$

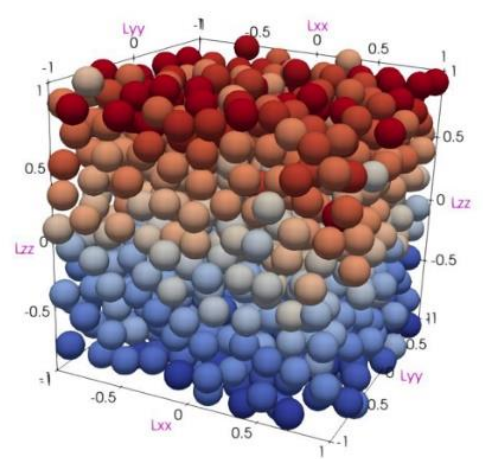

c) $\gamma_{x z}=5.0$
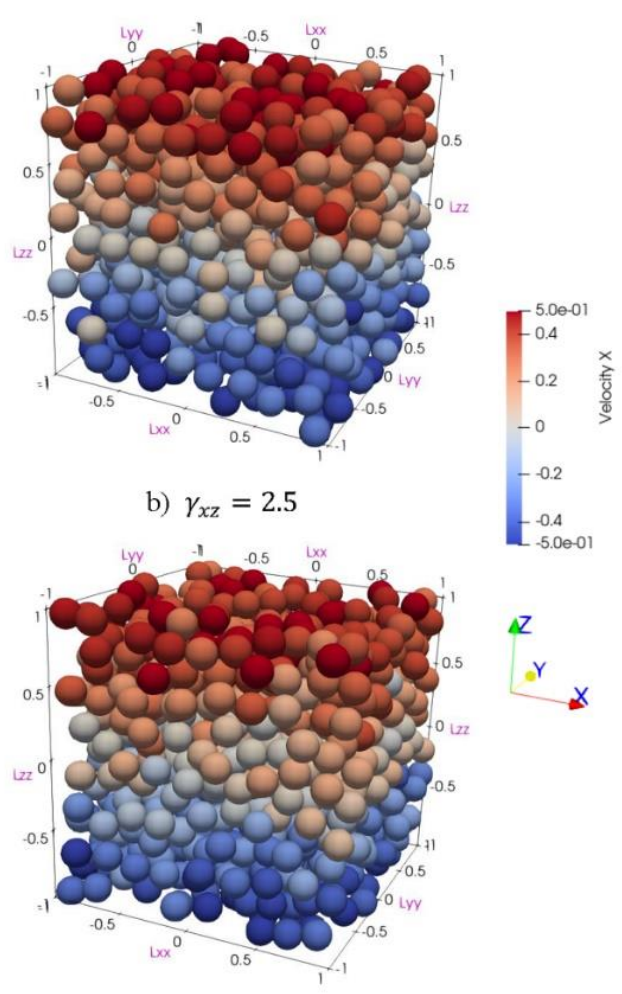

d) $\gamma_{x z}=10$

Fig. 6 Snapshots of RVE model at different shear deformation stages 


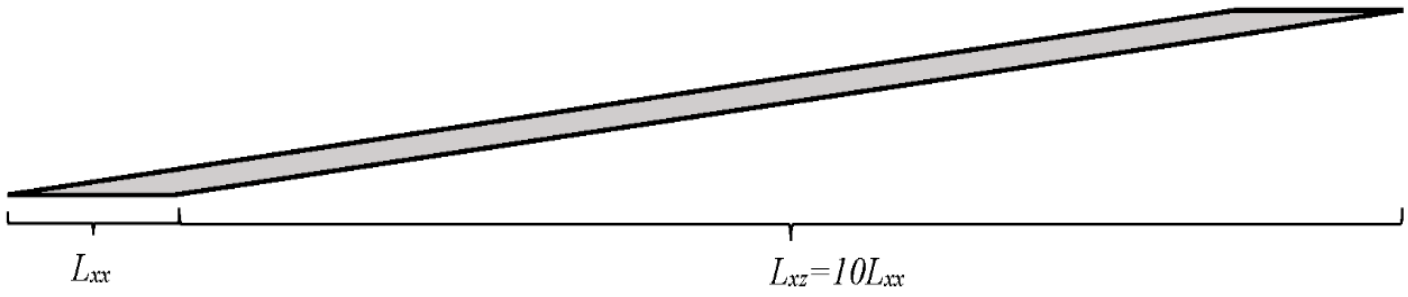

Fig.7 Large deformation of the conventional RVE box

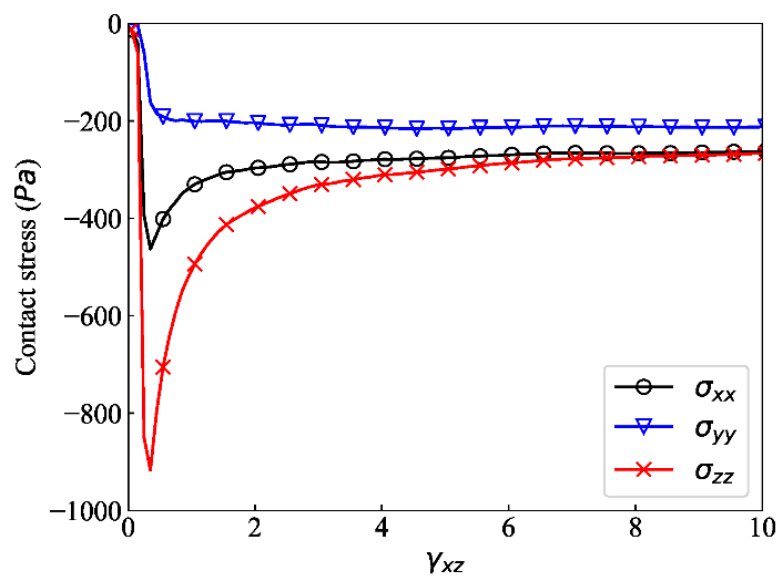

a) diagonal components

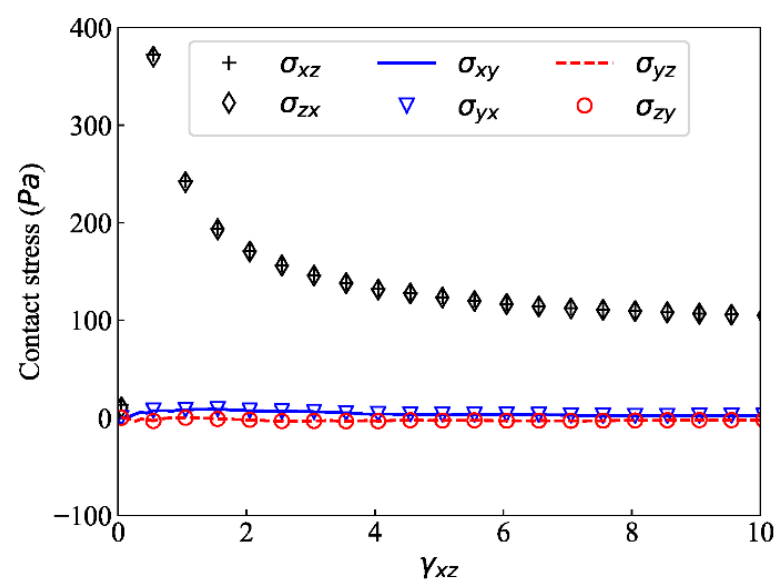

b) off-diagonal components

Fig. 8 Variations of contact stress $(n=0.548)$ 


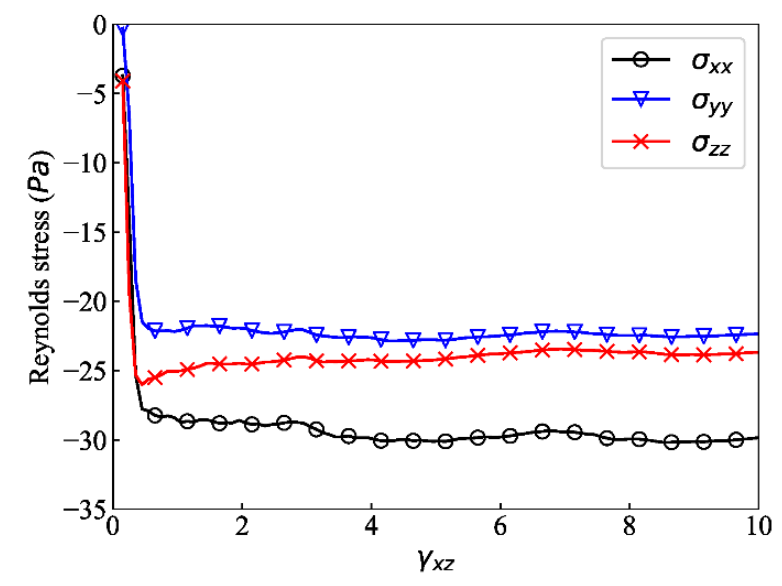

Fig. 9 Variation of Reynolds stress $(n=0.548)$

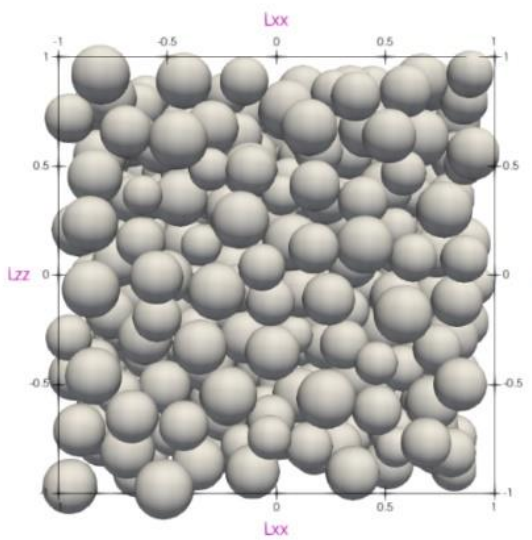

a) $t=0.0 \mathrm{~s}$

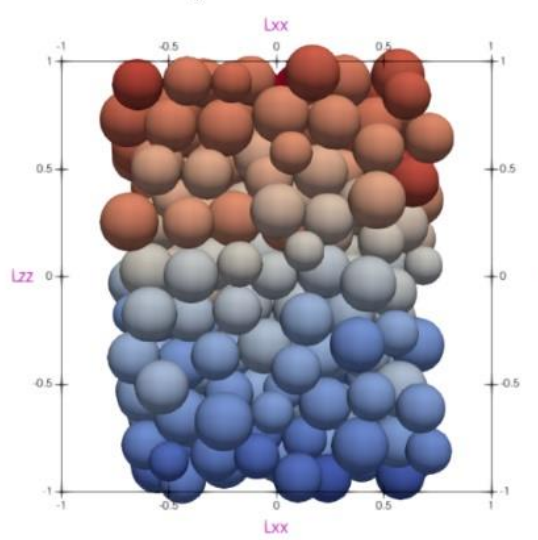

c) $t=10.0 \mathrm{~s}$

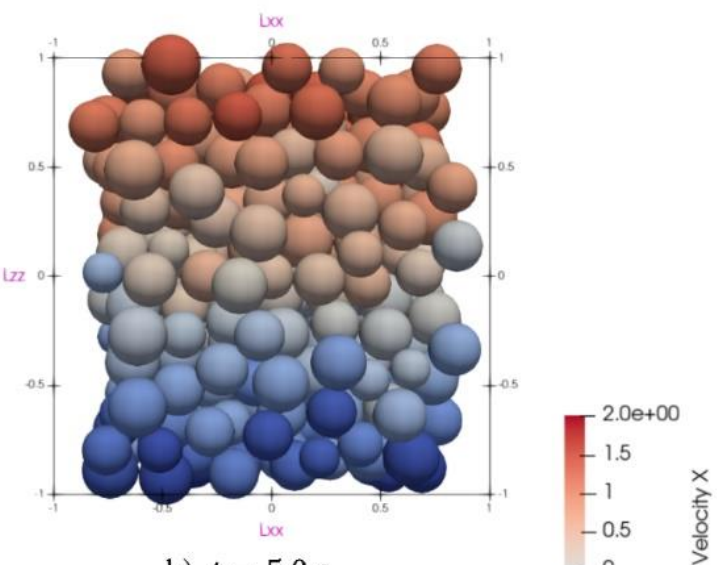

b) $t=5.0 \mathrm{~s}$

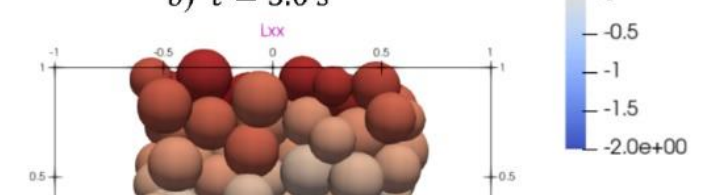

$-2.0 e+00$

Fig. 10 Snapshots of RVE model with complex loading at different instants 


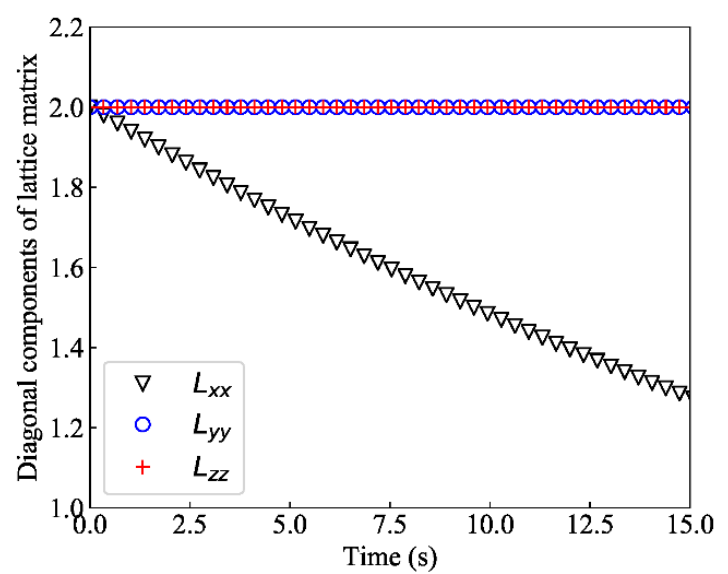

Fig. 11 Variation of $L_{x x}, L_{y y}, L_{z z}$

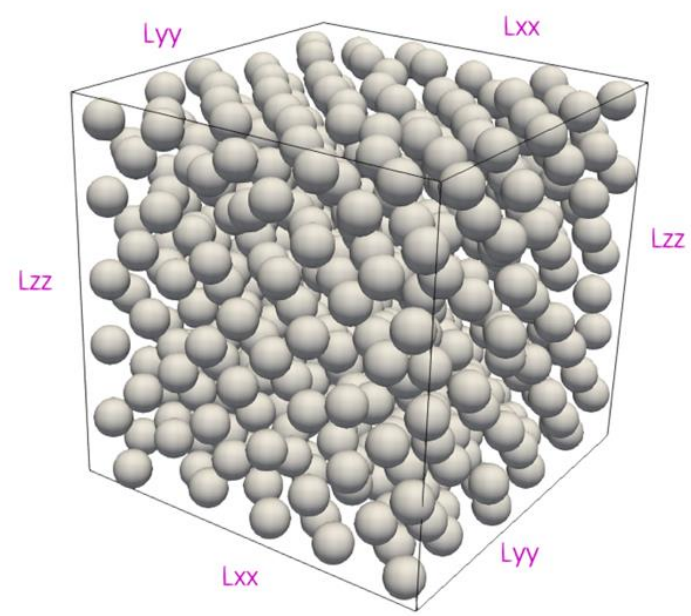

a) loose $(n=0.738)$

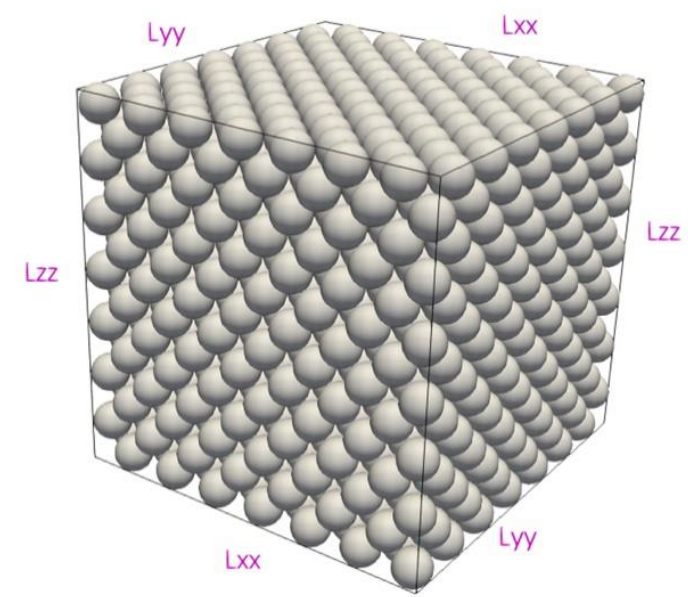

b) dense $(n=0.282)$

Fig. 12 Two granular packings with different porosities 


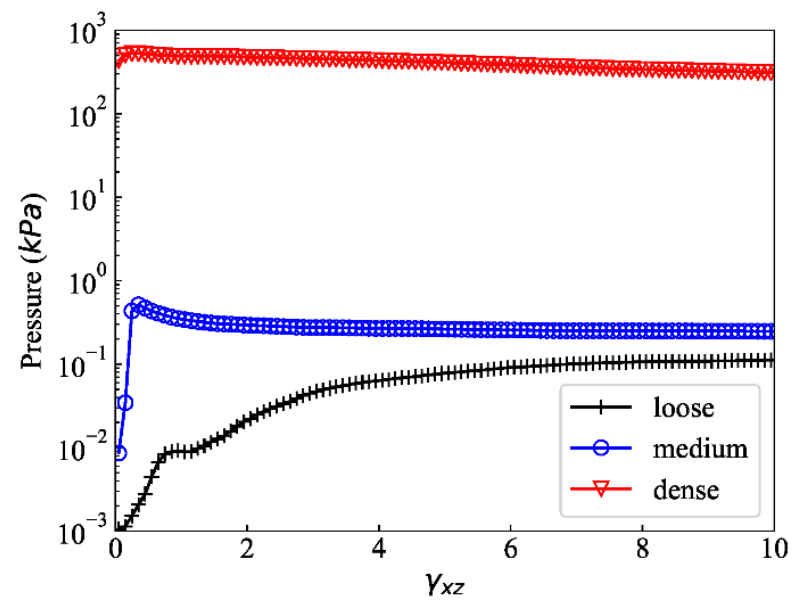

Fig.13 Comparison of hydrostatic pressure

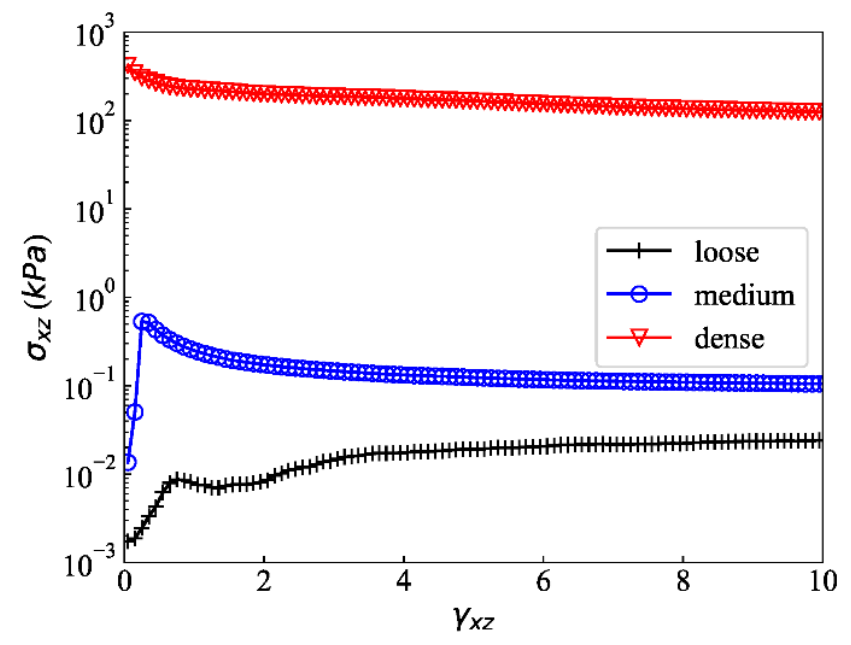

Fig.14 Comparison of off-diagonal component $\sigma_{x z}$

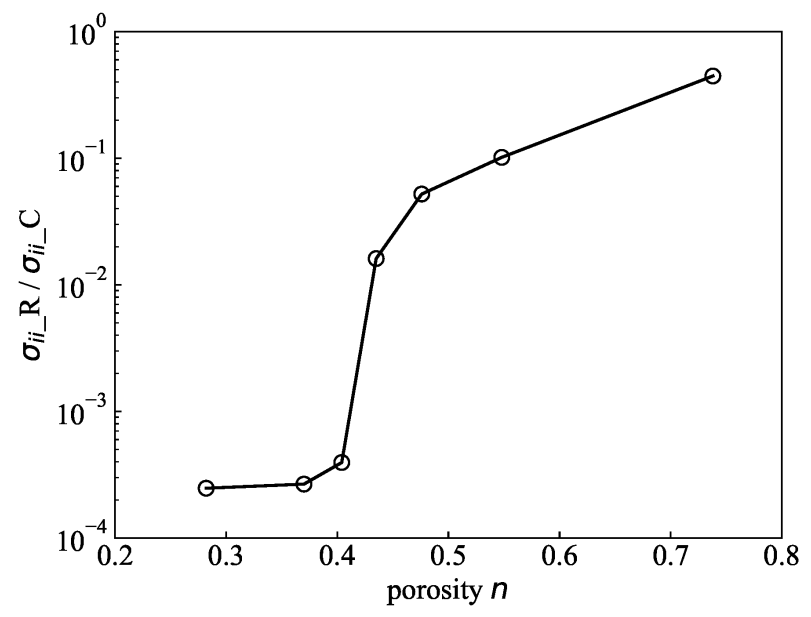

Fig. 15 Ratio of trace of Reynolds stress to trace of contact stress 